\title{
Multidrug resistant microorganisms causing neonatal septicemia: In a tertiary care hospital Lahore, Pakistan
}

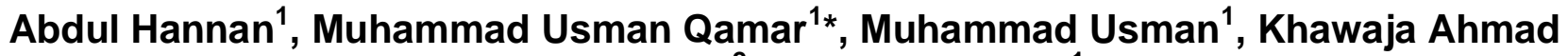 \\ Irfan Waheed ${ }^{2}$ and Kanwal Rauf ${ }^{1}$ \\ ${ }^{1}$ Department of Microbiology, University of Health Sciences, Lahore-Pakistan. \\ ${ }^{2}$ Department of Neonatology, the Children Hospital, Lahore-Pakistan.
}

Accepted 26 April, 2013

\begin{abstract}
Septicemia is an important cause of morbidity and mortality in neonates. Appropriate clinical diagnosis and empirical treatment is crucial as pathogens causing sepsis and their antibiotic susceptibility pattern varies in different settings. Therefore, this study was designed to determine the pattern of organisms causing neonatal sepsis and their antimicrobial resistance profile. One hundred and three blood culture specimens were collected aseptically. The specimens were processed in BACTEC 9120. Antimicrobial resistance profile was determined using Kirby-bauer disc diffusion method. Isolates showing resistance to any of the third generation cephalosporins were further confirmed for the production of ESBL by double disk diffusion method according to CLSI 2009 guidelines. Of 103 blood cultures, $71(68.9 \%)$ were positive. Out of these positive cultures, $62(87 \%)$ cases grew pure growth while $9(12 \%)$ cases had polymicrobial infection. Out of these eighty isolates $30(37.5 \%)$ and $50(62.5 \%)$ were found in early and late onset septicemia, respectively. Thirty $(42.2 \%)$ neonates died and $K$. pneumoniae was the main causative agent. The predominant isolates were $K$. pneumoniae $(n=40)$ and CoNS $(n=11)$. More than $90 \%$ of Gram-negative isolates showed resistance against $\beta$-lactams, coamoxiclav and amikacin. More than $\mathbf{5 0 \%}$ of Gram-positive isolates were resistant to penicillin, macrolides, ciprofloxacin and co-trimoxazole. ESBLs producing $K$. pneumoniae $(n=19)$, MRCoNS $(n=6)$, MRSA $(n=4)$ have also been found in this study. The study concludes that frequency of organisms was mainly found in late-onset neonatal septicemia. Multidrug resistant and ESBL producing organisms were main contributing factor towards high mortality.
\end{abstract}

Key words: Neonatal septicemia, antimicrobial resistance (AMR), ESBL.

\section{INTRODUCTION}

Neonatal Septicemia (NS) has been documented as a leading cause of mortality and morbidity all over the world (Qazi and Stoll, 2009). World Health Organization (WHO) reported over 4 million neonatal deaths occur each year globally; 3 million of these deaths occur in early neonatal period. Mortality rate of NS has been more prevalent in developing countries which account $98 \%$ of deaths in neonates (Oestergaard et al., 2011; WHO, 2006). UNICEF (2009) reported that more than 500 neonates die daily in Pakistan and mortality rate is $54 / 1000$ live 
births (LBs). About $40 \%$ of these deaths are due to infections and asphyxia. Amongst Asian countries, Pakistan has the eighth highest rate of newborns deaths (Moccia et al., 2009). To establish and diagnose NS, it has been documented by a positive blood culture in the first month (0 to 28 days) of life (Gheibi et al., 2008).

NS has two types, early-onset neonatal sepsis (EONS) and late-onset neonatal sepsis (LONS); both are defined as illness appearing from birth to seven days and from eight to twenty-eight days postnatal respectively (Oestergaard et al., 2011; Gheibi et al., 2008). Various causative factors have been reported for transmission of these infections and which are related to maternal risk factors in early period and birth canal acquisition. On the other hand, late-onset of infections is acquired from home or hospital environment (Zaidi et al., 2005). Most common causes of deaths in neonatal period are infections $(32 \%)$ including septicemia, meningitis, pneumonia, diarrhea and neonatal tetanus, followed by birth asphyxia and injuries (29\%) and prematurity (24\%) (Liu et al., 2012). Overall, Gram-negative bacteria are more frequent causes of NS.

Commonly isolated micro-organisms include Klebsiella spp., Escherichia coli, Pseudomonas spp., Salmonella spp., Staphylococcus aureus, coagulase negative staphylococci (CoNS), Streptococcus pneumoniae, Group B streptococci and S. pyogene (Oestergaard et al., 2011; Muhammad et al., 2010; Gheibi et al., 2008). For better management and control of these infections conventional antibiotics had remained important in practice. For last three decades, these agents have been found useless in combating the causative organisms ultimately led to the resistance. Antimicrobial resistance (AMR) is a growing problem worldwide and it is estimated that approximately 50 to $60 \%$ of more than two million nosocomial infections in the USA each year are caused by AMR bacteria (Jones, 2001). In developing countries, AMR resulted in extra financial burden, prolongation of hospital stay, and devastating or even fatal consequences (Byarugaba, 2004). According to food and drug administration (FDA), approximately $\$ 21$ to 34 billion is attributable to treat infections due to AMR pathogens in USA (Spellberg et al., 2011). It has become the dire need to document and investigate these resistant isolates in different populations so that appropriate measures can be taken to overcome the neonatal infections.

In developing countries like Pakistan, there is lack of proper microbiological diagnostic facilities. Therefore most of the physicians prescribe antibiotics to treat neonatal septicemia on empirical grounds. Using of these antibiotics dimly, might exploring the new ways of various drug resistance by the microorganism. Besides antibiotics prescribed are broad spectrum which can leads to more resistance among micro-organisms (Rasul et al., 2007). The present study was investigated to explore the frequency of microbes, mortality rates and antimicrobial susceptibility pattern of blood culture isolates in neonatal septicemia from a tertiary care hospital, Lahore, Pakistan.

\section{METHODOLOGY}

A total of 103 neonates (age: 0 to 28 days of life) suspected for septicemia were selected for this study and blood samples were collected from a neonatology unit of the children hospital Lahore, Pakistan from April to July 2009, by aseptic technique. Pediatric blood culture bottles (Bactec Peds plus/F) were inoculated with 1 to $3 \mathrm{ml}$ of blood and incubated in BACTEC 9120 instrument (Becton Dickinson, USA) for at least 5 days before declaring negative.

\section{Identification/purification of isolates}

Positive specimens were sub-cultured on Blood agar and MacConkey agar and incubated at $35^{\circ} \mathrm{C}$ for $24 \mathrm{~h}$. The isolates were preliminary identified on the basis of morphology and cultural characteristics. Gram-positive isolates were biochemically identified by catalase, slide and tube coagulase and DNase test. Whereas, Gram-negative isolates were biochemically identified by cytochrome oxidase and confirmed by API 20E and 20NE (BioMerieux France). Reference strains, S. aureus ATCC (25923), E. coli ATCC (25922), $P$. aeruginosa ATCC (27853) and $E$. faecalis ATCC (29212) were included to monitor quality control.

\section{Antimicrobial susceptibility testing}

Antimicrobial susceptibility of isolates was performed by KirbyBauer disk diffusion method using Mueller-Hinton agar (Oxoid UK), according to Clinical Laboratory Standards Institute (CLSI) 2009 guidelines (Wilker et al., 2009). The plates were prepared and incubated at $35^{\circ} \mathrm{C}$ for $24 \mathrm{~h}$. Implanted antibiotics were penicillin (10 $\mu \mathrm{g})$, ampicillin $(10 \mu \mathrm{g})$, cefoxatin $(30 \mu \mathrm{g})$, co-amoxiclav $(20 / 10 \mu \mathrm{g})$, cefuroxime $(30 \mu \mathrm{g})$, ceftriaxone $(30 \mu \mathrm{g})$, ceftazidime $(30 \mu \mathrm{g})$, cefepime $(30 \mu \mathrm{g})$, erythromycin $(15 \mu \mathrm{g})$, clindamycin $(2 \mu \mathrm{g})$, vancomycin $(30 \mu \mathrm{g})$, linezolid $(30 \mu \mathrm{g})$, ciprofloxacin $(5 \mu \mathrm{g})$, amikacin $(\mu \mathrm{g})$, co-trimoxazole $(25 \mu \mathrm{g})$, imipenem $(10 \mu \mathrm{g})$, and meropenem (10 $\mu \mathrm{g})$. The interpretation of susceptibility results were done according to CLSI guidelines (Wikler et al., 2009). Statistical analysis was done using SPSS 16.0

\section{RESULTS}

Out of 103 blood cultures, $71(68.9 \%)$ were found to be positive. Out of these positive cultures, $62(87 \%)$ cases grew pure growth while $9(12 \%)$ cases had polymicrobial infections. Out of these eighty isolates $30(37.5 \%)$ and 50 $(62.5 \%)$ were found in early and late onset septicemia respectively. Neonatal mortality rate was 30 (42.2\%); 12 neonates were died in EONS and 18 were died in LONS. $K$. pneumoniae was the predominant pathogen in both EONS and LONS and also responsible for $21(70 \%)$ neonatal deaths.

Gram-negative rods were more frequent than Grampositive bacteria with a frequency of $58(72.5 \%)$ and 22 $(27.5 \%)$ respectively. K. pneumoniae $(n=40)$ was the commonest pathogen followed by CoNS $(n=11)$, Staphylococcus aureus $(\mathrm{n}=6)$ and Sphingomonas paucimobilis $(n=5)$ (Table 1$)$. Most of the Gram-negative 
Table 1. Frequency of blood isolates $(n=80)$ from neonatal septicemia.

\begin{tabular}{lccc}
\hline Isolates & Distribution $(\mathbf{n})$ & EONS ( $\mathbf{n}=\mathbf{3 0})$ & LONS ( $\mathbf{n}=\mathbf{5 0})$ \\
\hline Gram-negative rods $(\mathrm{n}=58)$ & 40 & 10 & 30 \\
Klebsiella pneumoniae & 5 & 4 & 1 \\
Sphingomonas paucimobilis & 3 & 1 & 2 \\
Escherichia coli & 2 & 1 & 1 \\
Enterobacter cloacae & 2 & 1 & 1 \\
Burkholderia cepacia & 2 & 1 & 1 \\
Acinetobacter baumannii & 1 & 1 & 0 \\
Klebsiella ornitholytica & 1 & 1 & 0 \\
Flavomonas oryzihabitans & 1 & 0 & 0 \\
Proteus penneri & 1 & 1 & \\
Pseudomonas aeruginosa & & & 7 \\
Gram-positive cocci (n=22) & & & 3 \\
Coagulase negative staphylococci & 11 & 3 & 3 \\
Staphylococcus aureus & 6 & 0 & 0 \\
Enterococcus faecalis & 3 & 2 & \\
Viridians streptococci & 2 & & \\
\hline
\end{tabular}

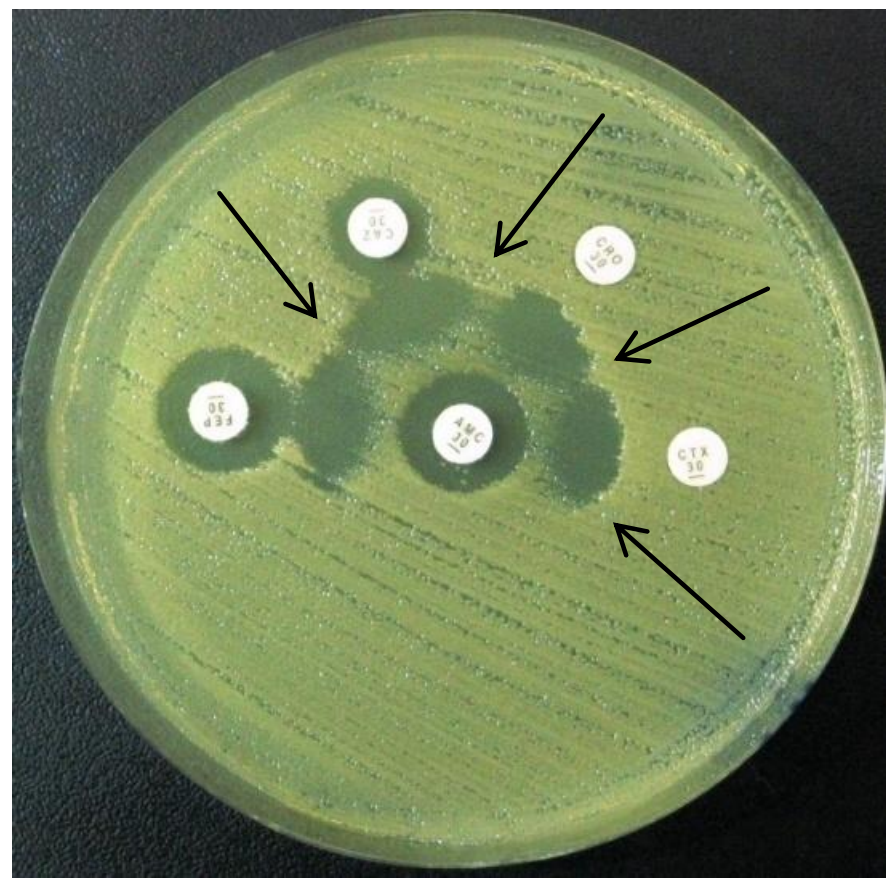

Figure 1. Demonstration of ESBL phenomenon. Black arrows indicate the enlarged zone of inhibition of each the CTX, CAZ, $\mathrm{CRO}$ and FEP disks which placed at $20 \mathrm{~mm}$ distance from AMC disk. AMC; Co-amoxiclav, CTX; Cefotaxime, CAZ; Ceftazidime, CRO; Ceftriaxone, FEP; Cefepime.

rods showed $100 \%$ resistance against commonly used antibiotics, the high resistance was mainly due to ESBL producing $K$. pneumoniae among gram negative isolates $(n=19)$ (Figure 1). Most effective antibiotics against Gram-negative rods were carbapenems (imipenem, meropenem) (Table 2). Antibiotic resistance profile of 
Table 2. Resistant pattern of Gram-negative bacteria.

\begin{tabular}{|c|c|c|c|c|c|c|c|c|c|c|}
\hline Antimicrobials & $\begin{array}{c}\text { K. pneumoniae } \\
(n=40) \%\end{array}$ & $\begin{array}{c}\text { S. paucimobilis } \\
(n=5) \%\end{array}$ & $\begin{array}{l}\text { E. coli } \\
(n=3) \%\end{array}$ & $\begin{array}{c}\text { E. cloacae } \\
(n=2) \%\end{array}$ & $\begin{array}{l}\text { B. cepacia } \\
(n=2) \%\end{array}$ & $\begin{array}{c}\text { A. baumannii } \\
(n=2) \%\end{array}$ & $\begin{array}{c}\text { K. ornitholytica } \\
(n=1) \%\end{array}$ & $\begin{array}{c}\text { F. oryzihabitans } \\
(n=1) \%\end{array}$ & $\begin{array}{l}\text { P. penneri } \\
(\mathrm{n}=1) \%\end{array}$ & $\begin{array}{l}\text { P. aeruginosa } \\
(n=1) \%\end{array}$ \\
\hline Ampicillin & 100 & 100 & 100 & 100 & 100 & 100 & 100 & 100 & 100 & 100 \\
\hline Co-amoxiclave & 100 & 100 & 100 & 100 & 100 & 50 & 100 & 100 & 100 & 100 \\
\hline Cefuroxime & 100 & 100 & 100 & 100 & 100 & 50 & 100 & 100 & 100 & 100 \\
\hline Ceftriaxone & 100 & 100 & 100 & 100 & 50 & 50 & 100 & 100 & 100 & 100 \\
\hline Ceftazidime & 100 & 100 & 100 & 100 & 100 & 50 & 100 & 100 & 100 & 100 \\
\hline Cefepime & 40 & 100 & 100 & 100 & 50 & 50 & 100 & 100 & 100 & 100 \\
\hline Amikacin & 100 & 0 & 100 & 100 & 100 & 50 & 100 & 100 & 100 & 100 \\
\hline Ciprofloxacin & 47.5 & 100 & 100 & 100 & 0 & 50 & 100 & 0 & 0 & 100 \\
\hline Co-trimoxazole & 60 & 100 & 100 & 100 & 0 & 50 & 100 & 0 & 100 & 100 \\
\hline Imipenem & 0 & 100 & 0 & 100 & 0 & 0 & 0 & 100 & 0 & 100 \\
\hline Meropenem & 2.6 & 100 & 0 & 0 & 0 & 0 & 0 & 100 & 0 & 100 \\
\hline
\end{tabular}

Gram-positive cocci showed more than $50 \%$ of CoNS and $S$. aureus were resistant to penicillin, co-trimoxazole, and clindamycin with MRCoNS $(n=6)$ and MRSA $(n=4)$ but susceptible to vancomycin and linezolid (Table 3). Overall all the Gram-negative rods were found resistant to ampicillin, ceftazidime followed by co-amoxiclav and cefuroxime $(98 \%)$, ceftriaxone and cefepime $(97.1 \%)$ and amikacin $(91.2 \%)$ (Figure 2). More than $80 \%$ of Gram-positive cocci were resistant to penicillin, ampicillin and erythromycin and were susceptible to vancomycin and linezolid (Figure $3)$.

\section{DISCUSSION}

Septicemia is a leading cause of deaths in neonates particularly in developing countries (Muhammad et al., 2010). Emerging of AMR has further aggravated the problem. In 103 investigated cases, there were 71 (68.9\%) positive. Out of these positive cultures, 80 different organisms including both Gram-positive and Gram-negative were isolated which is in accordance with the previous studies done in India, Pakistan and Bangladesh (Gyawali and Sanjana, 2012; Khan et al., 2012; Monjur et al., 2010). Some of the cases were also having polymicrobial infection. This might be due to poor hygienic status in hospitals. Mortality rate observed was $42.2 \%$ which is also highlighted in another study from Pakistan (Asim Khurshid, 2005). The causative agent for these neonatal deaths was $K$. pneumonia, which accounted for $70 \%$ of the deaths.

Neonatal septicemia is more prone to late-onset infections. The present study showed LONS was more common than EONS, which is in contrast to some previous studies from Iran and Pakistan (Gheibi et al., 2008; Aurangzeb and Hameed, 2003). The increased EONS might be due to the fact that they have not been given prophylactic drugs, prematurity and low birth weight of neonates. However, another finding from India was in support of the present study (Sundaram et al., 2009). This is more related with nosocomial infections.

In present study K. pneumoniae from Gramnegative rods and CoNS from Gram-positive cocci were commonly isolated in EONS and LONS. In contrary to our investigations, some other studies from Pakistan described E. coli, S. aureus (Waheed et al., 2003), Klebsiella and Moraxella spps found in EONS and LONS (Muhammad et al., 2010). This could be due to the presence of Gram-negative rods in hospital environment. Based on our results, other studies have also reported comparable findings for these commonly isolated $K$. pneumoniae and CoNS in Gramnegative rods and Gram-positive cocci respectively from Pakistan, India, Iran and UK (Aletayeb et al., 2011; Ghoutaslour and Nahaeimr, 2007; Butt et al., 2006).

In contrast to the current results, another study from Pakistan reported Enterobacter spp (52\%) 
Table 3. Resistant pattern of Gram-positive cocci.

\begin{tabular}{lcccc}
\hline Antimicrobials & CoNS $(\mathbf{n}=\mathbf{1 1}, \%)$ & S. aureus $(\mathbf{n}=\mathbf{6}, \%)$ & E. faecalis $(\mathbf{n}=\mathbf{3}, \%)$ & V. streptococci $(\mathbf{n}=\mathbf{2}, \%)$ \\
\hline Penicillin & 72.7 & 83.3 & 100 & 100 \\
Ampicillin & $\mathrm{NT}$ & $\mathrm{NT}$ & 66.6 & 100 \\
Cefoxitin & 54.5 & 66.6 & $\mathrm{NT}$ & $\mathrm{NT}$ \\
Erythromycin & 90.1 & 66.6 & 66.6 & 100 \\
Clindamycin & 60 & 50 & $\mathrm{NT}$ & $\mathrm{NT}$ \\
Amikacin & 0 & 16.6 & 100 & 100 \\
Ciprofloxacin & 27.3 & 66.6 & 100 & 50 \\
Co-trimoxazole & 63.6 & 66.6 & 50 & 100 \\
Vancomycin & 0 & 0 & 0 & 0 \\
Linezolid & 0 & 0 & 0 & 0 \\
\hline
\end{tabular}

NT, Not tested.

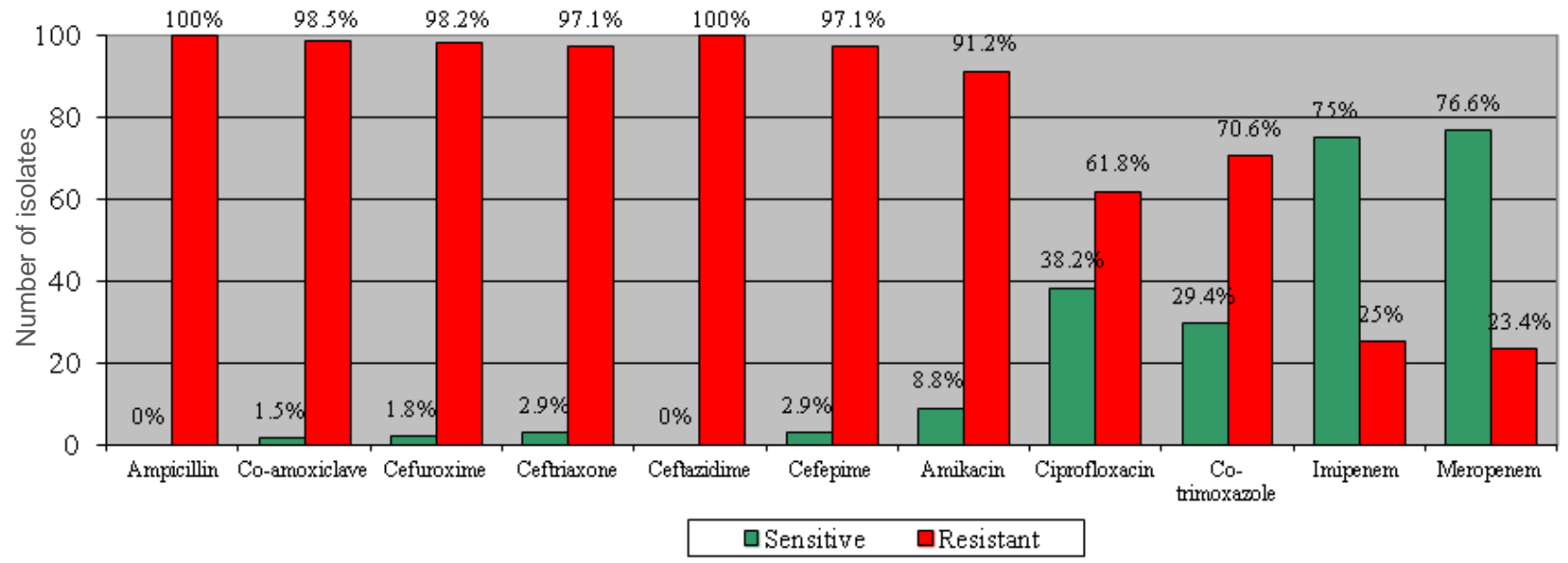

Figure 2. Overall susceptibility pattern of Gram-negative rods.

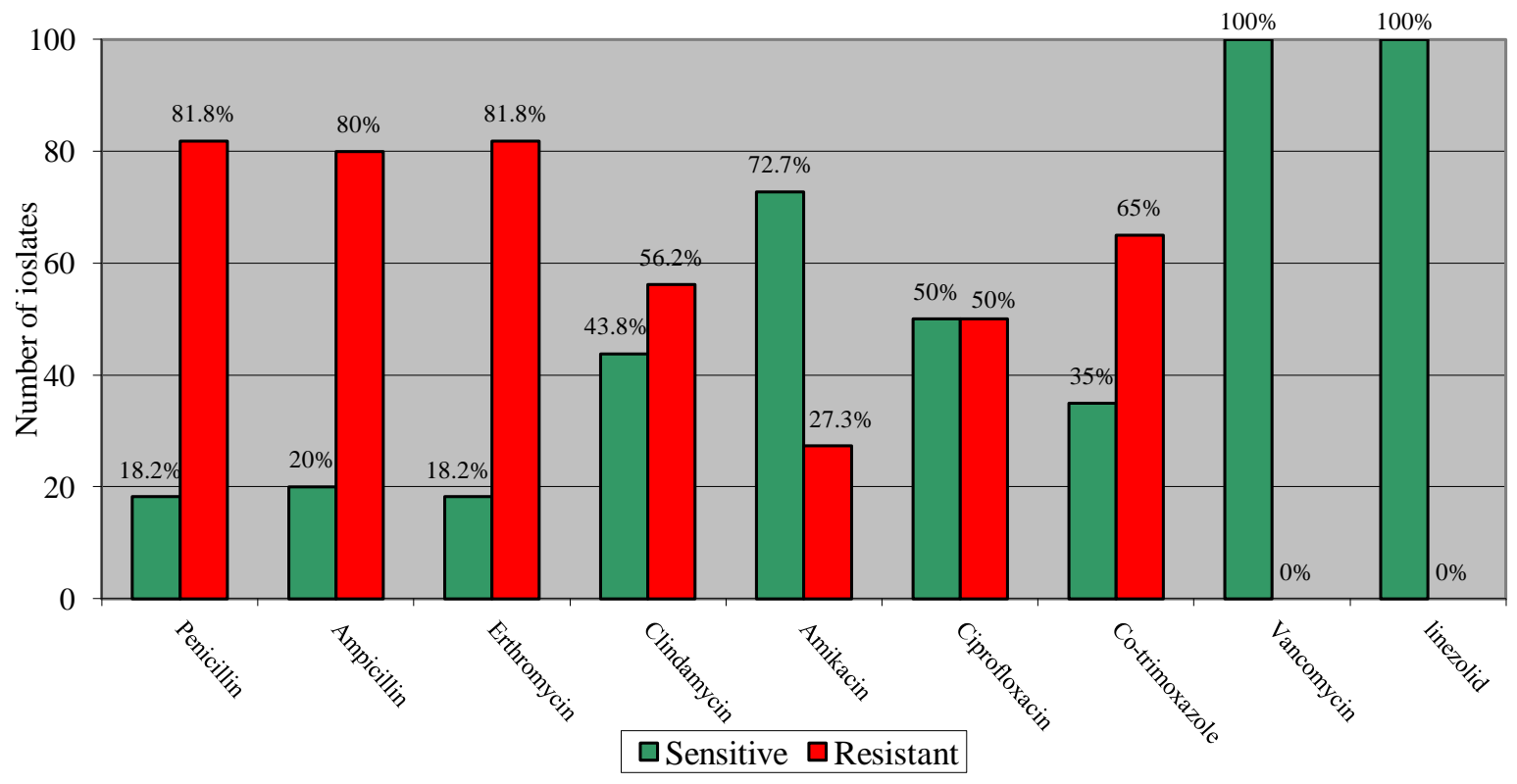

Figure 3. Overall percent susceptibility pattern of Gram-positive micro-organisms. 
and E. coli (30\%) as the leading pathogens (Akhter and Raza, 2005). This difference might be due to their geographical distribution of organisms.

In this study, antibiotic resistance profile of Gramnegative rods is shown in Table 2. K. pneumoniae was the most resistant pathogen among Gram-negative rods. Findings of this study are in accordance with the previous studies showing more than $70 \%$ of K. pneumoniae and $E$. coli resistant to various antibiotics in India and Pakistani hospital (Saleem et al., 2012; Movahedian et al., 2006). WHO also have reported the high incidence of AMR against common pathogens including $(K$. pneumoniae, $E$. coli, E. cloacae and S. aureus) in NS (Oestergaard et al., 2011). In contrary to this study some other researcher documented low to moderate resistance in Gramnegative rods (Mhada et al., 2012; Butt et al., 2006). Among drug resistant isolates, ESBLs producing pathogens have been reported in different region of the world. We have reported $23.7 \%$ ESBLs producing $K$. pneumoniae (Figure 3 ), which is almost comparable with the previous investigation described in NS (Abdel-Hady et al., 2008; Damjanovai et al., 2005).To best of our knowledge this is the first study reporting $K$. pneumoniae as ESBLs producing pathogen in NS from Pakistan.

Gram-positive isolates including MRCoNS $(n=6)$ and MRSA $(n=4)$ are similar to previous data in hospitalized neonates (Cordero et al., 1999). Antimicrobial resistant pattern of Gram-positive cocci is illustrated in Table 3. More than $50 \%$ of Gram-positive isolates were resistant routine antibiotics except vancomycin and linezolid (Figure 3). Various other studies have also highlighted the frequently isolated pathogens including $S$. aureus and CoNS (Khan et al., 2012; Muhammad et al., 2010; Thaver et al., 2009). Spread of MDR is mainly linked to an inappropriate infection control practices. Various other factors seriously contribute to the spread of bacterial resistance including contaminated intravenous catheters, feeding tube and various environmental surfaces (door handles, sucker machine, incubators, mattresses, wash basins, floor, sink, emergency trolley, ventilator, ambo bag, laryngeal scopes) and colonized hands of staff. Nurseries are often seriously overcrowded and understaffed, sharing of baby beds (two to three babies in a cot). Substandard sterilization and disinfection practices are common. We also observed lack of standard practices such as preparation of medication in contaminated area, reuse of ambo bag, ventilator and laryngeal scopes to other neonates without disinfection. It is well documented that neonates have immature immune system and unable to provide defense against virulent pathogens. Premature babies are at high risk because of lack of protective maternal antibodies, underdeveloped innate immunity and fragile, easily damaged skin (Zaidi et al., 2005). Another major factor to acquire resistance in our setup is irrational use of empirical therapy which is not according to the WHO criteria (Oestergaard et al., 2011).
It is concluded from the current study that AMR is imparting very crucial role in the spread of neonatal infections. These resistant bugs are being spread from the hospitals to the community. Now, it is the need of hour to improve infection control practices, irrational use of antibiotics should be avoided and empirical treatment regimen should be revisited to prevent further resistance.

\section{ACKNOWLEDGEMENTS}

We are grateful to University of Health Sciences, Lahore Pakistan for financial and logistic support for this research project. We also acknowledge Neonatology Department of The Children Hospital, Lahore, Pakistan for providing clinical material.

\section{REFERENCES}

Abdel-hady $\mathrm{H}$, Hawas S, El-daker M, El-kady R (2008). Extendedspectrum beta-lactamase producing Klebsiella pneumoniae in neonatal intensive care unit. J. Perinatol. 28:685-90.

Akhter RHA, Raza R (2005). Neonatal sepsis in NICU: bacterial isolates and their sensitivity pattern. J Surg. Pak. 10:18-21.

Aletayeb $\mathrm{SMH}$, Khosravi AD, Dehdashtian M, Kompani F, Mortazavi SM, Aramesh MR (2011). Identification of bacterial agents and antimicrobial susceptibility of neonatal sepsis: A 54-month study in a tertiary hospital. A. J. Microbiol. Res. 5(5):528-531.

Asim khurshid JR (2005). Neonatal mortality in a district hospital setup. Ann. King Edward Med. Coll. 11:373-6.

Aurangzeb B, Hameed A (2003). Neonatal sepsis in hospital-born babies: bacterial isolates and antibiotic susceptibility patterns. J. Coll. Physic. Surg. Pak. 13:629-32.

Butt TKRA, Farooqi R, Zafar A, Shah AA (2006). Blood culture and sensitivity pattern in Neonatology unit of Children Hospital Lahore. Ann. King Edward Med. Coll. 12:79-81.

Byarugaba DK (2004). A view on antimicrobial resistance in developing countries and responsible risk factors. Int. J. Antimicrob. Agents 24:105-10.

Cordero L, Sananes M, Ayers LW (1999). Bloodstream infections in a neonatal intensive-care unit: 12 years' experience with an antibiotic control program. Infect. Control Hosp. Epidemiol. 20:242-6.

Damjanovai ORZ, Toth A, Petro IC, Szikra L, Paszti J (2005). Epidemiology and infection control of ESBL producing Klebsiella pneumoniae strains caused 5 outbreaks in a Hungarian neonatal intensive care unit during the years 2001-2004. Clin. Microbiol. Infect. 11.

Gheibi S, Fakoor Z, Karamyyar M, Khashabi J, Ilkhanizadeh B, Sana FA, Mahmoodzadeh $H$, Majlesi AH (2008). Coagulase Negative Staphylococcus; the Most Common Cause of Neonatal Septicemia in Urmia, Iran. Iran J. Pediatr. 18:237-243.

Ghoutaslour GZ, Nahaeimr (2007). Klebsiella pneumoniae in neonatal sepsis: A 3-years-study in the Pediatric Hospital of Tabriz, Iran. Jpn. J. Infect. Dis. 60:126-128.

Gyawali N, Sanjana RK (2012). Bacteriological Profile and Antibiogram of Neonatal Septicemia. Indian J. Pediatr. 5:1-5.

Jones RN (2001). Resistance patterns among nosocomial pathogens Trends over the past few years. Chest 119(2):397-404.

Khan MA, Khan A, Shah F, Munir A (2012). Neonatal sepsis: a study of causative pathogens and their antimicrobial sensitivity pattern at tertiary hospital. Gomal J. Med. Sci. 10:244-247.

Liu L, Johnson HL, Cousens PS, Perin J, Scott S, Lawn JE (2012). Global, regional, and national causes of child mortality: an updated systematic analysis for 2010 with time trends since 2000 . The Lancet. 379:2151-2161.

Mhada TV, Fredrick F, Matee MI, Massawe A (2012). Neonatal sepsis 
at Muhimbili National Hospital, Dar es Salaam, Tanzania; aetiology, antimicrobial sensitivity pattern and clinical outcome. BMC Pub. Health 12:1-6.

Moccia P, Anthony D, Brazier C, Noia M, Gebre-egziabher H, Goodman E (2009). State of the World. Maternal and Newborn Health-A Pakistani Perspective. UNICEF unit for children.URL: http://www.unicef.org/publications/files/SOWC 2009 Main Report 03112009.

Monjur F, Rizwan F, Asaduzzaman M, Nasrin N, Ghosh NK, Apu AS (2010). Antibiotic sensitivity pattern of causative organisms of neonatal septicemia in an urban hospital of Bangladesh. Indian J. Med. Sci. 64:265-71.

Movahedian AH, Moniri R, Mosayebi Z (2006). Bacterial culture of neonatal sepsis. Iran. J. Pub. Health 35:84-89.

Muhammad Z, Ahmed A, Hayat U, Wazir MS, Rafiyatullah, Waqas H (2010). Neonatal sepsis: causative bacteria and their resistance to antibiotics. J. Ayub Med. Coll. Abbottabad 22:33-35.

Oestergaard MZ, Inoue M, Yoshida S, Mahanani WR, Gore FM, Cousens S (2011). Neonatal Mortality Levels for 193 Countries in 2009 with Trends since 1990: A Systematic Analysis of Progress, Projections and Priorities. PLoS Med. 8:1-13.

Qazi SA, Stoll BJ (2009). Neonatal sepsis: a major global public health challenge. Pediatr. Infect. Dis. J. 28:1-2.

Rasul CH, Hassan MA, Habibullah M (2007). Neonatal sepsis and use of antibiotic in a tertiary care hospital. Pak. J. Med. Sci. 23:78-81.

Saleem AF, Qamar F, Zaidi AK (2012). Review of Incidence and Antibiotic Susceptibility Pattern of Klebsiella pneumoniae Neonatal Sepsis in a Neonatal ICU of Karachi, Pakistan. 30th Annual Meeting of the European Society For Paediatric Infectious Diseases. Thessaloniki, Greece.
Spellberg B, Blaser M, Guidos RJ, Boucher HW, Bradley JS, Eisenstein BL (2011). Combating antimicrobial resistance: policy recommendations to save lives. Clin. Infect. Dis. 52(5):397-428.

Sundaram VKP, Dutta S, Mukhopadhyay K, Ray P, Gautam V (2009). Blood Culture Conformed Bacterial Sepsis in Neonates in a North Indian Tertiary Care Centre: Change over the Last Decade. Jpn. J. Infect. Dis. 62:46-50.

Thaver D, Ali SA, Zaidi AKM (2009). Antimicrobial Resistance Among Neonatal Pathogens in Developing Countries. Pediatr. Infect. Dis. J. 28:19-21.

Waheed M, Laeeq A, Maqbool S (2003). The etiology of neonatal sepsis and patterns of antibiotic resistance. J. Coll. Physic. Surg. Pak. 13:449-52.

WHO (2006). Neonatal and Perinatal Mortality Country, Regional and Global Estimates. URL: http://whqlibdoc.who.int/publications/2006/9241563206_eng

Wikler MA, Cockerill FR, Craig WA, Dudley MN, Eliopoulos GM, Hecht DW (2009). Clinical and Laboratory Standards Institute (CLSI) 19th Informational Supplement. 29(3):M02-A10.

Zaidi AK, Huskins WC, Thaver D, Bhutta ZA, Abbas Z, Goldmann DA (2005). Hospital-acquired neonatal infections in developing countries. Lancet 365:1175-88. 